\title{
MIGRACIONES CALIFICADAS: EL CASO DE LAS COLOMBIANAS EN BUENOS AIRES
}

\author{
Skilled migration: the case of Colombian women in Buenos Aires
}

\author{
Alessandra Ciurlo ${ }^{7}$ \\ Diana Couto-Mármora² \\ Mónica Santagata ${ }^{3}$
}

\begin{abstract}
Resumen. Este artículo nace de la observación de un pequeño grupo de jóvenes mujeres colombianas altamente calificadas, residentes en la Ciudad Autónoma de Buenos Aires. El análisis de orden cualitativo y desde una dimensión psicológica y social, afronta las trayectorias migratorias de dichas mujeres. Se examinan las motivaciones para emigrar de las jóvenes de donde emerge una fuerte necesidad de emancipación emocional y económica de la familia, emancipación social de la sociedad de origen, encubiertas en gran parte bajo la búsqueda de oportunidades de estudio y profesionales. En el estudio se analizan además, algunos factores de estrés presentes en la experiencia migratoria, la incidencia de la familia y las presiones de orden social que ejerce la sociedad de origen. Se indaga cómo durante el proceso migratorio se van consolidando entre las entrevistadas, la exploración y apropiación de nuevos modos de vida, cómo se van delineando cambios en las relaciones interpersonales, en el ejercicio de los roles sociales y en las relaciones de género.
\end{abstract}

Palabras clave: mujer migrante, migración calificada, migración por estudio, género, familias transnacionales, migración colombiana, Argentina.

\begin{abstract}
This article stems from the observation of a small group of highly qualified young women, who reside in the Autonomous City of Buenos Aires. A qualitative analysis of the migratory paths of these women is performed from a psychological and social dimension. An examination of the motivations for migration shows a strong need of emotional and economic emancipation of the family and social emancipation from the society of origin, mostly disguised in the search for academic and professional
\end{abstract}

1 Pontificia Universidad Gregoriana de Roma. Roma, Italia.

2 Universidad Tres de Febrero UNTREF. Buenos Aires, Argentina.

3 Programa de Asistencia Médica Transcultural del Hospital Italiano. Buenos Aires, Argentina. 
opportunities. The study also analyzed some stress factors present in the migratory experience and the incidence of family and social pressures exerted by the society of origin. The study inquires how new explorations and appropriation processes of new forms of life are consolidated among those interviewed during the migration process and how new interpersonal relationships take shape in the exercise of social roles and gender relations.

Keywords: migrant woman, skilled migration, student migration, gender, transnational families, Colombian migration, Argentina.

\section{Introducción}

Este artículo pretende abordar algunas dinámicas y fenómenos de las migraciones femeninas, específicamente de jóvenes mujeres colombianas calificadas residentes en la Ciudad Autónoma de Buenos Aires. Se trata de una categoría particular que forma parte del colectivo de colombianos residentes en Argentina y que ha crecido de manera consistente en los últimos años, suscitando gran interés y curiosidad de parte de varios actores sociales tanto en Colombia como en Argentina.

El grupo objeto de análisis es poco visible ya que se trata de mujeres calificadas bien integradas culturalmente y laboralmente en la sociedad bonaerense y con altos niveles de satisfacción en lo que respecta a su proyecto migratorio. Pertenecen a clases sociales acomodadas en Colombia y comparten una fuerte pero a la vez velada motivación de emancipación en sus proyectos migratorios; motivación encubierta, en la mayor parte de los casos, bajo razones de tipo profesional o académico que las estimulan a concebir la decisión de migrar y poner en práctica el proyecto migratorio. El estudio nace de la observación de las diversas motivaciones que este grupo de mujeres demuestra tanto en la dimensión socio-cultural como en la dimensión psico-emotiva del proceso migratorio.

Los interrogantes que tienen la función de motor del proceso investigativo giran alrededor de cuáles son las motivaciones que llevan a las migrantes a distanciarse de la familia de origen y a permanecer años en un contexto diferente del propio y con algunas restricciones económicas y qué factores de estrés afrontan. Además, cuál es el lugar que ocupa la distancia geográfica y afectiva en sus vidas, cuáles son las relaciones transnacionales que mantienen y cuáles las funciones que tales relaciones vienen a adoptar. Igualmente, qué factores de estrés están presentes en el proceso migratorio, cuáles las diferentes formas de emancipación social, afectiva y de género, y si la migración se configura como una forma de resolución de la adolescencia o, dicho en otras palabras, como un rito de paso hacia la vida adulta.

La primera parte del artículo se focaliza en el marco teórico, con un abordaje interdisciplinario que integra elementos de los ámbitos sociológico y 
psicológico, para seguir con la nota metodológica en la que se define el diseño de la investigación. Sucesivamente se afronta la contextualización del problema de estudio, para finalizar con el análisis de los hallazgos y algunas conclusiones que de ellos se desprenden.

\section{Marco teórico}

En los últimos decenios el escenario de las migraciones internacionales ha visto un número cada vez mayor de mujeres migrantes, de hecho su presencia ha igualado la de hombres, aunque en algunos flujos ha alcanzado proporciones más altas. Los motivos que inducen a las mujeres a emigrar son diversos; es un hecho que gran parte de ellas lo hace de manera independiente y autónoma, si bien la migración en algunos casos puede ser parte de estrategias familiares que tienen como objetivo el bienestar del núcleo familiar ${ }^{4}$. Es cierto también que una parte de las mujeres migra con un proyecto de tipo individual, por ejemplo en aras de su carrera profesional o de aumentar su capital humano, o como una búsqueda personal en la dimensión emocional, entre otras. Empero, el análisis de las migraciones femeninas se ha focalizado principalmente en la familia, en particular en el rol reproductivo y productivo de las mujeres que tienen en ella, y en las dinámicas de cuidado en un contexto global ${ }^{5}$. En el ámbito laboral, el estudio se ha centrado en sectores de baja calificación como el trabajo doméstico y el de cuidado a la persona ${ }^{6}$, dada la enorme demanda de mano de obra en algunos países de destino debido, en parte, a las carencias del Estado social de Bienestar ${ }^{7}$. En cambio, como sostiene Kofman ${ }^{8}$, las migraciones de mujeres calificadas no han gozado de suficiente atención ya que la migración calificada se ha relacionado principalmente con algunos sectores del ámbito laboral que se consideran típicamente "masculinos". Entre ellos, la economía del conocimiento, la ciencia, las nuevas tecnologías de la información y la comunicación, la finanza y la gerencia, que además se consideran como promotores de la creación de la riqueza global. No se ha tenido en cuenta la participación de las mujeres en tales sectores y tampoco su presencia significativa en sectores como la salud, el trabajo social, el estudio, la investigación científica, menospreciando también el valor e importancia que dichas esferas tienen en la vida económica y social de los países de origen y destino.

\footnotetext{
4 HONDAGNEU SOTELO, Pierrette, AVILA, Ernestine. I'm here, but I'm there: The meaning of Latin transnational motherhood; OSO, Laura. L'immigration en Espagne des femmes chefs de famille.

5 HOCHSCHILD, Arlie R. Global care chains and emotional surplus value.

6 PARREÑAS, Rachel. Servants of globalization: Woman migration and domestic work; PARELLA, Sònia. La maternidad a distancia de las empleadas domésticas latinoamericanas en España. La vulneración del derecho a la vida familiar en el contexto de la internacionalización de la reproducción.

7 AMBROSINI, Maurizio. Dentro il welfare invisibile: aiutanti domiciliari immigrate e assistenza agli anziani.

8 KOFMAN, Eleonore. Gender and skilled migration in Europe, p. 63, 69.
} 
Algunos estudiosos demuestran sin embargo que en las migraciones calificadas las mujeres son más numerosas que los hombres -tanto en los países en vía de desarrollo como en los desarrollados- y emigran siendo estudiantes, trabajadoras, refugiadas o esposas de migrantes. En algunos casos, entre las razones para emigrar, influyen algunos factores de género, como pueden ser el acceso diferenciado al mercado de trabajo en sus países de origen y las discriminaciones que allí sufren por ser mujeres ${ }^{9}$. El género influye también en la inserción en el nuevo contexto, siendo usual que las migrantes sufran procesos de descalificación, pues frecuentemente no logran obtener un trabajo en el sector en el que se formaron o se ven abocadas a realizar trabajos por debajo de su calificación ${ }^{10}$. No obstante las dificultades en el campo laboral, desarrollan diversas estrategias para integrarse en la sociedad de destino, demostrando con ello su capacidad de agencia ${ }^{11}$.

En lo que concierne a la migración por estudio, según Riaño y Piguet $^{12}$ en su reseña bibliográfica sobre las investigaciones en este campo conceptual, son diversos los factores que influyen en la decisión de emigrar y alrededor de los cuales se ha profundizado en el análisis. Entre ellos tienen un rol importante no sólo las estrategias que las personas construyen evaluando los costos y beneficios para la adquisición de capital humano, sino también las políticas migratorias nacionales y las políticas públicas respecto a la educación y la formación (tanto en la sociedad de origen como en la de destino). Respecto de la experiencia migratoria de las y los estudiantes en las sociedades de acogida, en los diversos estudios se analiza su experiencia allí, e igualmente las perspectivas emergentes laborales que tiene la población estudiantil, las posibilidades de movilidad posterior al periodo de estudio y las posibilidades de retorno al país de origen.

Como en otros tipos de migración, también en el que acá es objeto de estudio emergen formas de vida y experiencias transnacionales ${ }^{13}$ dados los vínculos que las y los estudiantes mantienen y además crean entre la sociedad que los acoge y aquella de la que parten. La cultura de origen, lejos de desaparecer, permanece viva, transmitiéndose en gran parte a través de la familia y las relaciones que se mantienen entre los parientes. Una familia que asume una connotación transnacional, ya que vive separada entre las fronteras por un periodo de tiempo más o menos largo, pero sigue funcionando como

9 DOCQUIER, Frédéric et alii. Are skilled women more migratory than skilled men?; RAGHURAM, Parvati. Situating women in the brain drain discourse: Discursive challenges and opportunities.

10 KOFMAN, op. cit.; 2012; RIAÑO, Yvonne, BAGHDADI, Nadia, WALST WATER, Doris. Les migrantes qualifiées et leurs possibilités d'intégration professionnelle en Suisse.

${ }^{11}$ RIAÑO, Yvonne. Minga biographic workshops with highly skilled migrant women: Enhancing spaces of inclusion.

12 RIAÑO, Yvonne, PIGUET, Etienne. International student migration.

${ }^{13}$ BASH, Linda, GLICK-SCHILLER, Nina, SZANTON-BLANC, Cristina. Toward a Transnational Perspective on Migration. 
familia, manteniendo las relaciones a distancia entre sus miembros, que se siguen sintiendo parte de una unidad y percibiendo su bienestar desde una perspectiva colectiva ${ }^{14}$. Son familias, como sugiere Merla ${ }^{15}$, en las que se da un intenso intercambio de cuidados en las dos direcciones de la migración: en la de quien se va y en la de quien se queda.

Hay que tener en cuenta, sin embargo, que la familia además de sufrir muchos cambios es un espacio de negociación y de lucha entre sus miembros, más que un espacio de armonía e igualdad ${ }^{16}$. Es igualmente una unidad de reproducción y transmisión cultural, también de los sistemas y relaciones de género que determinan desigualdades entre hombres y mujeres.

La observación desde una perspectiva psicológica de los cambios en la configuración, funcionamiento, roles y posiciones en la familia, pero también los cambios que derivan de la misma migración entre los migrantes, permite advertir que estos pueden tener implicaciones más o menos profundas en el equilibrio psicológico de cada persona. Es usual que se presenten situaciones de estrés y que aparezcan factores estresores que acompañan al migrante durante su proceso.

Para comprender cómo inciden dichos factores en la vida del migrante resulta útil adoptar el modelo que desarrolla Lazarus ${ }^{17}$ acerca del estrés, modelo centrado en los procesos cognitivos que se desarrollan en torno a una situación estresante.

Desde este modelo, la definición de una situación como molesta, inoportuna, inadecuada, innecesaria, amenazante o peligrosa por parte de la persona es motivo suficiente para que un determinado conjunto de variables ambientales provoquen estrés. El estrés nace básicamente en los procesos cognitivos que se desarrollan alrededor de una situación estresante; estos derivan en primer lugar de las evaluaciones que hace la persona del estresor $y$, en segundo lugar, de los recursos personales, sociales o culturales disponibles que en ese momento tiene para hacer frente a la situación de estrés. La definición de una situación de estrés radica entonces en la relación específica que, en un momento y un contexto determinados, se establece entre la persona y la variable o situación ambiental.

Las estrategias de afrontamiento para resolver el estrés pueden tomar principalmente dos direcciones: asumir comportamientos o actos dirigidos a gestionar la fuente de estrés o, en cambio, orientados a provocar una variación

\footnotetext{
${ }^{14}$ BRYCESON, Deborah, VOURELA, Ulla (eds.). The transnational family. New European frontiers and global networks.

${ }^{15}$ MERLA, Laura. La circulación de cuidados en las familias transnacionales.

${ }^{16}$ KABEER, Naila. Benevolent dictators, maternal altruists and patriarchal contracts: Gender and household economics.

${ }^{17}$ LAZARUS, Richard S. Stress and emotion. A new synthesis.
} 
en la forma como es percibida y vivida la situación de estrés, regulando de manera más efectiva las reacciones emocionales negativas.

\section{Nota metodológica}

De acuerdo con las preguntas iniciales que dan impulso a este estudio exploratorio -el cual se configura como la primera fase de una investigación de mayor envergadura sucesiva-, la aproximación elegida se inscribe en el paradigma cualitativo.

En el diseño de la pesquisa se eligió abordar el problema de investigación mediante la perspectiva etno-sociológica de Bertaux ${ }^{18}$, de acuerdo con la cual no se recogen datos para verificar hipótesis formuladas con anterioridad, sino para descubrir las características y el funcionamiento de un contexto social desconocido y por determinar en su especificidad. Ello con la finalidad de elaborar interpretaciones plausibles más que explicaciones causales de los fenómenos estudiados.

El instrumento utilizado para la recolección de los datos es la entrevista en profundidad, siguiendo las pautas de las "Historias de vida" de Sautu19, mediante el cual se focaliza la atención en un particular segmento de la historia del sujeto ligado a eventos específicos, como es, en este caso, la migración.

La muestra de esta fase preliminar de la investigación está compuesta por 8 mujeres residentes en la Ciudad Autónoma de Buenos Aires, elegidas mediante la técnica de muestreo subjetivo por elección razonada siguiendo algunas características específicas, lo que las define además, como testigos privilegiados de la fase posterior del estudio. Del tipo de muestra y su tamaño se deduce un cierto sesgo que no permite que la muestra sea considerada representativa de la población de mujeres colombianas residentes en dicha ciudad, y tampoco se puede proceder a la generalización de los resultados.

Las entrevistas ${ }^{20}$ fueron realizadas durante los meses de mayo y junio de 2016, fueron grabadas y transcritas con el consentimiento de las entrevistadas que participaron en el estudio voluntariamente, y a quienes se garantizó el anonimato.

\section{Contextualización del problema de investigación}

\section{La migración colombiana}

Los flujos migratorios desde Colombia, que han alcanzado volúmenes significativos en los últimos cincuenta años, se han caracterizado por involucrar un elevado número de personas en diferentes oleadas. Se calcula que

\footnotetext{
${ }^{18}$ BERTAUX, Daniel. Racconti di vita. La prospettiva etnosociologica.

19 SAUTU, Ruth (comp.). El método biográfico. La reconstrucción de la sociedad a partir del testimonio de los actores.

${ }^{20}$ Las entrevistadoras fueron Diana Couto-Mármora y Mónica Santagata.
} 
actualmente cerca del $10 \%$ de la población total de nacionales colombianos ${ }^{21}$ reside fuera del país.

Las principales metas de las migraciones en el continente americano han sido Estados Unidos y, en menor escala, Venezuela, Ecuador, México y Perú; y últimamente, los países del Cono Sur. Hacia Europa, los flujos, cuyos comienzos en la dimensión significativa señalada se remontan a los años 1970, aumentaron notablemente después del 11 de septiembre de 2001, esto debido al cierre de las fronteras estadounidenses. El destino más importante ha sido España, país ligado a Colombia por lazos históricos y por tener el mismo idioma; los colombianos presentes de manera estable allí son aproximadamente $400.000^{22}$. Y le siguen, en su orden, Italia, Francia e Inglaterra.

Con la crisis europea de 2008 y sus fuertes repercusiones en los países del Sur de Europa, los flujos que allí se dirigían, se han empezado a redireccionar hacia otras metas, entre las cuales algunos países de la misma región. De acuerdo con el Panorama Migratorio de América del Sur ${ }^{23}$, Colombia es el país con mayor número de emigrantes en Suramérica. Entre los países que se destacan como receptores de dicha emigración se hallan Chile, Brasil y Argentina, país que posee una gran tradición como receptor de migrantes, al punto de constituir un elemento significativo y aun determinante de su dinámica demográfica y su vida social, económica y cultural.

Los flujos migratorios procedentes de Colombia son profundamente heterogéneos y se han ido modificando a través del tiempo en virtud de circunstancias propias de los contextos y de las oportunidades emergentes. Las causas principales de los flujos han sido la difícil situación socio-económica del país durante diversas décadas, problemas de seguridad ligados al narcotráfico, el aumento de las tensiones políticas con la intensificación del conflicto armado. En efecto, los emigrantes parten principalmente en busca de mejores condiciones de vida, mayores oportunidades laborales, profesionales y de estudio, y en algunos casos, en busca de seguridad física. Es preciso aclarar que Colombia, además de tener una alta tasa de emigración, se caracteriza por desplazamientos internos de población de carácter masivo y por la migración "forzada" de refugiados y solicitantes de asilo ante los países limítrofes.

Otra de las peculiaridades de los flujos migratorios desde Colombia es la alta tasa de personas con formación terciaria ${ }^{24}$. Según datos del Banco Mundial de 2011, la tasa de emigración de capital humano altamente calificado

\footnotetext{
${ }^{21}$ Según el Ministerio de Relaciones Exteriores, en el año 2012 cerca de 4.700 .000 colombianos vivían en el exterior. Las estimaciones del DANE (Departamento Nacional de Estadística) con base en el Censo de 2005 dan como población total colombiana en 2014, 47.661.787 personas.

${ }^{22}$ OIM. Perfil migratorio de Colombia, 2010, p. 50.

${ }^{23}$ OIM. Panorama migratorio de América del Sur, p. 13.

${ }^{24}$ Ibidem, p. 33.
} 
corresponde al 10,4\% de la población colombiana, del cual el 5,7\% está constituido por médicos formados en el país. Entre los destinos principales de los colombianos con alta calificación se hallan España, Estados Unidos, Francia, Alemania, Brasil y Argentina ${ }^{25}$.

\section{Argentina como contexto de llegada de los flujos provenientes de Colombia}

La observación de la migración colombiana a Argentina permite advertir que, si bien no es muy numerosa, diversos indicadores ${ }^{26}$ señalan que desde el año 2006 ha tenido un aumento exponencial, llegando las y los colombianos a consolidarse como la décima mayor población de inmigrantes presente en su territorio. Según los últimos datos disponibles del Censo Nacional de Población Hogares y Viviendas de 2010, las y los colombianos en Argentina son 17.576, cifra que se sitúa a gran distancia de la de otras poblaciones latinoamericanas provenientes de los países limítrofes que, atraídas por las oportunidades laborales, se dirigen allí con mayor facilidad: es el caso de quienes provienen de Paraguay (550.713), Bolivia (345.272) y Chile (191.147), siendo asimismo relevante el número de inmigrantes provenientes de Perú (157.514).

Con base en los datos del Censo de 2010 se puede observar que las y los colombianos se concentran principalmente en la Ciudad de Buenos Aires (55\%) y en la Provincia de Buenos Aires (22\%), y en muy baja proporción en las provincias de Córdoba (5\%), Santa Fe (4\%) y Mendoza (3\%). La desagregación por sexo revela una leve disparidad entre hombres y mujeres a favor de estas últimas, que representan el 51,4\% del total. Es una población joven y en edad activa, la gran mayoría de la cual $(89,7 \%)$ tiene entre 15 y 64 años; un tercio del total (32,5\%) tiene entre 15 y 35 años.

Sin evidentes diferencias entre hombres y mujeres, el Censo de 2010 revela que se trata de una población calificada, con un alto capital humano. Más de la mitad cuenta con estudios universitarios de pregrado y postgrado, aunque las mujeres son más numerosas que los hombres en dicha categoría (el 60\% de las mujeres vs. el 56\% de los hombres). Cerca de un décimo (9,1\%) cuenta con estudios de tipo superior no universitario, y un quinto del total con una formación secundaria: en este caso son los hombres los más numerosos (el 23,3\% de los hombres vs. el 19,7\% de las mujeres). Melella ${ }^{27}$ sostiene que la población estudiantil está compuesta principalmente por estudiantes universitarios de áreas vinculadas a la publicidad, el cine, el diseño, las ciencias sociales y la salud.

\footnotetext{
${ }^{25}$ OIM. Perfil migratorio de Colombia, 2012, p. 75-76.

${ }^{26}$ Los datos de los diferentes censos nacionales y también las tendencias de las radicaciones de la población extranjera en Argentina.

27 MELELLA, Cecilia. Migraciones emergentes hacia la Argentina: colombianos y ecuatorianos. Breve panorama y estrategias de inserción cultural, p. 25.
} 
Según los datos de la encuesta realizada por $\mathrm{OIM}^{28}$ en colaboración con otras instituciones ${ }^{29}$, este flujo migratorio no sólo se caracteriza porque la población concernida cuenta con altos niveles de estudio, sino porque gran parte de esta tiene la intención de continuar con su formación académica y su capacitación. Muchos inician sus carreras en Argentina y muchos otros se inscriben en maestrías y doctorados en universidades públicas y privadas. De hecho, más de la mitad de las y los colombianos viajan a Argentina con la intención de estudiar (56,8\%); de allí que su radicación en el país no sea permanente sino prevalentemente temporal. El resto de la población se distribuye de manera bastante equilibrada entre quien se instala en Argentina por motivos laborales $(13,5 \%)$-generalmente profesionales- y quien lo hace por motivos familiares $(11,5 \%)$. También hay quien va a estudiar y trabajar simultáneamente $(10,2 \%)$.

Es pertinente anotar que las razones por las que un consistente número de estudiantes universitarios emigra de Colombia están estrechamente relacionadas con sus políticas públicas educativas y con la visión mercantil de la educación que deriva del modelo neoliberal imperante. Según un estudio realizado por el Sistema Universitario Estatal $^{30}$, en las últimas dos décadas se ha puesto en marcha un proceso de desfinanciamiento estructural de las universidades públicas, lo que ha significado un recorte en los programas de bienestar, en la inversión de infraestructura, un aumento en la subcontratación y en la prestación de servicios externos, y paralelamente, también, el aumento del valor de las matrículas de licenciaturas y postgrados.

Argentina, por su lado, como contexto de arribo, se ha consolidado como polo receptor de flujos regionales de estudiantes. Di Lorenzo ${ }^{31}$ sostiene que el aumento significativo de estudiantes extranjeros en las universidades argentinas en los últimos años responde a distintos factores causales, entre los que figuran: una política estatal intensiva de promoción del sistema educativo; un incremento en los convenios de cooperación entre las universidades de la región en el marco de la internacionalización de la educación superior; un tipo de cambio favorable en un período determinado; y por último, aunque no menos importante, la existencia de universidades públicas y de mayor calidad en términos relativos a nivel regional. Argentina ofrece un sistema universitario desarrollado -público y privado- a costos bajos y/o con diversas facilitaciones y acceso a beneficios para los estudiantes, mediante los convenios internacionales instituidos para el efecto.

\footnotetext{
${ }^{28}$ OIM. La migración colombiana..., op. cit.

${ }^{29}$ Con una muestra compuesta por 1.561 colombianos que residen en Argentina.

30 SISTEMA UNIVERSITARIO ESTATAL. Desfinanciamiento de la educación superior en Colombia.

${ }^{31}$ DI LORENZO, Delfina. El Estado argentino frente al fenómeno de la migración de estudiantes universitarios de origen latinoamericano (2003-2011), p. 8.
} 
Retornando a los colombianos residentes en Argentina, respecto de su situación ocupacional, aunque esta presenta características disímiles y heterogéneas, la encuesta $\mathrm{OIM}^{32}$ revela que se trata de una migración altamente calificada ya que el $89 \%$ del total se distribuye en tres grandes grupos: profesionales científicos (42\%), personal de apoyo administrativo (33\%) y técnicos y profesionales de nivel medio (14\%). Además, cerca de tres cuartas partes de quienes trabajan (72\%) logran insertarse en un sector que contribuye a la construcción de su experiencia profesional, además de tratarse de trabajos prevalentemente desarrollados en condiciones de contratación formal.

$\mathrm{OIM}^{33}$ señala, con relación a la vida social, que la población colombiana residente en Argentina es relativamente estable e integrada ya que dos quintos tiene residencia permanente $(42,6 \%)$ y un tercio la tiene de tipo temporal $(33,6 \%)$; menos de un quinto (17\%) tiene una residencia "precaria".

Según Puente ${ }^{34}$, gran parte de quienes emigran por razones de estudio, una vez finalizada la formación, deciden volver a la tierra natal, si bien en algunos casos emigran hacia otros destinos; en esto pesan la falta de oportunidades laborales en Argentina, los vínculos familiares en el país de origen, el hecho de conseguir emplearse en Colombia o de haber conservado el trabajo que tenían antes de partir. Los datos de la encuesta OIM $^{35}$ revelan que la mitad de la población entrevistada quiere retornar a Colombia (50\%) y dos quintas partes (41\%) se quieren quedar en Argentina.

\section{Resultados}

\section{El perfil de las entrevistadas}

Las mujeres que participaron en el estudio son personas jóvenes que tienen entre 22 y 40 años, están en edad activa, y por el momento del ciclo vital en el que se encuentran podrían formar una pareja y tener hijos. La mayoría, sin embargo, viven solas, aunque a su llegada a Argentina hayan compartido la vivienda con otras personas (conocidos y/o amigos o parientes). Se observa la tendencia ampliamente mayoritaria a postergar la conformación de un núcleo familiar, aunque tres de ellas vive con su pareja y en una situación afectiva estable.

En general se trata de profesionales -la mayoría en el área de la saludcon un nivel de educación alto, algunas poseen estudios de pregrado y otras de posgrado y doctorales. Muchas de ellas trabajan y estudian al mismo tiempo.

\footnotetext{
32 OIM. La migración colombiana..., op. cit., p. 54-55.

33 Ibidem, p. 22, 46, 79.

34 PUENTE, M. Cuando migrar es la opción. Estudiantes colombianos en Argentina: motivos y experiencias en sus trayectorias migratorias y estudiantiles.

35 OIM. La migración colombiana..., op. cit., p. 33.
} 
Es de notar que en el momento de la entrevista, la mayoría estaban cursando estudios de alto nivel. Gran parte de ellas son bilingües y provienen de escuelas y universidades de elevado reconocimiento en Colombia, siendo de origen socio-económico medio-alto y alto.

Las razones por las que se desplazan a Argentina están ligadas principalmente al estudio, de hecho muchas de ellas se dirigen allí por las oportunidades que encuentran para continuar con su trayectoria académica, pero también con su carrera profesional. Algunas se radican en Argentina específicamente porque en Colombia la retribución que reciben es baja y es difícil mejorarla, teniendo en cuenta, entre otras cosas, que el mercado laboral en su país de origen está en parte condicionado por estereotipos de género que limitan las posibilidades de desempeño y condicionan el reconocimiento profesional y económico de las mujeres. Se nota un cierto espíritu de aventura y sacrificio, ya que cuando se establecen en Argentina, algunas se insertan en trabajos de baja calificación que nunca realizarían en su país de origen, mientras se adaptan y logran una posición laboral más acorde con su perfil.

Algunas de estas colombianas han podido aprovechar oportunidades como becas y otras facilitaciones que les han dado la ocasión de ahondar en campos de estudio en los que en Colombia hubiera sido más difícil hacerlo, ya sea porque no los hay, porque el acceso a ellos es limitado y difícil, o porque resulta demasiado costoso. Es de notar también que dos de las entrevistadas se han desplazado a Argentina, y más explícitamente a Buenos Aires, con el proyecto de seguir a su pareja, aunque ello, en los dos casos, con la idea de desarrollar un proyecto personal, ya sea que el mismo esté enmarcado a la vez en el proyecto de la pareja o que sea independiente de ella.

\section{El deseo de emancipación}

En la decisión de emigrar generalmente hay otros factores concomitantes que se mezclan; entre ellos, y se trata de un factor que emerge con especial fuerza, está el deseo de emancipación de una sociedad que gran parte de las entrevistadas consideran conservadora, elitista, gobernada por estereotipos que dejan poco espacio a lo nuevo y a lo diferente. Si bien no todas las entrevistadas provienen del mismo contexto geográfico, coinciden en afirmar que la sociedad colombiana es fuertemente estratificada, regida por esquemas sociales muy estrictos que determinan comportamientos definidos, a los cuales no es fácil contraponerse y que cercenan la propia libertad. Estos esquemas en parte están relacionados con el rol social tradicional de las mujeres; no es casual que socialmente se espere de ellas que se ocupen de sus familias, de sus padres o de sus hermanos cuando falta la figura materna o una figura femenina que asuma este rol. Son frecuentemente emplazadas a ocupar un rol de cuidadoras y a asumir roles parentizados. Ante este cambio de posición 
familiar algunas se rebelan, ya que se sienten atrapadas y asfixiadas, una situación vital que con frecuencia las empuja a pensar en partir para evadirse de esta. Entre las entrevistadas, sin embargo, hay quien en su trayectoria de vida transita por diversas renuncias en aras de la familia, pero se advierte también que la movilidad internacional gira alrededor de la necesidad de establecer una distancia con una familia experimentada como absorbente, posesiva y machista, lo que conduce inevitablemente a situaciones de conflicto y a la búsqueda de soluciones que pueden incluir, una vez más, el proyecto de establecerse en el exterior.

La migración en este caso ayuda a algunas mujeres a desarrollar una perspectiva de vida y un proyecto personal no supeditado al núcleo familiar. Las ayuda a hacer una vida fuera de las normas y las expectativas sociales y familiares. En este contexto, la atribución de la opción de migrar a motivos de estudio o a razones profesionales les facilita la salida de la esfera familiar de manera pacífica. Ello sin que los padres perciban al alcance de su decisión, cuando es, en cambio, parte de un proceso de rebeldía y de ruptura que comienza antes del hecho de la migración.

La migración representa para algunas de las entrevistadas la posibilidad de salir o alejarse de la familia para buscar un espacio de independencia y de mayor autonomía en pos de modelos de vida diferentes. El hecho es que en las familias es frecuente que las relaciones sean muy estrechas e intensas, con una fuerte carga emocional; se trata usualmente de relaciones y vínculos simbióticos, y de los cuales resulta arduo desprenderse. Varias de las entrevistadas provienen de familias estresantes donde los padres ejercen una tracción endogámica hacia sus hijas, afectando de alguna manera, a menudo intensa, su deseo de libertad y su proyecto de vida personal. Bajo estas circunstancias, la migración es uno de los caminos para reducir el estrés y el sufrimiento psíquico poniendo distancia espacialmente, al no poder hacerlo en el plano emocional. Se trata de algo extremadamente necesario y útil en el proceso de desarrollo individual y de independización psicológica y personal.

Esto no significa sin embargo que dejen de extrañar a la familia, varias se sienten muy ligadas a su núcleo familiar de origen y se comunican con este cotidianamente, utilizando los nuevos medios de comunicación (chat, mensaje de texto, telefonía de bajo o ningún costo), en especial, la aplicación informática WhatsApp les permite estar en contacto con personas o con grupos. Por este medio se da un intercambio constante de información, impresiones, sentimientos e ideas acerca de la vida cotidiana. Todo ello produce una sensación de cercanía y ahonda las relaciones transnacionales, que brindan a las migrantes un apoyo fundamental en su vida en la sociedad de destino, ayudándolas a superar dificultades y pesares, a asumir sacrificios, a enfrentar la pérdida de estatus social y otras pérdidas. Las familias se configuran como 
un refugio y un centro de referencia emocional muy importante que ofrece diversos tipos de cuidado y respaldo a las migrantes. Las relaciones familiares son sin embargo contradictorias, porque brindan apoyo pero exigen también lealtades y formas de dependencia difíciles de manejar.

En la distancia algunas mujeres pueden atribuir un nuevo valor a la familia de origen, algunas la idealizan de manera que los conflictos existentes antes de emigrar parecen cristalizarse. Poner distancia física no parece ser siempre decisivo en la resolución de un conflicto familiar y afectivo, ya que las dinámicas disfuncionales se presentan una y otra vez, además de que no dejan de permear las actitudes de fondo en las nuevas circunstancias. Sin embargo, la migración primero, y la gestión de los vínculos parentales entre fronteras, después, se convierten en un recurso útil para disminuir el estrés y el condicionamiento vital que supone para ellas, permitiéndoles crear un espacio para reelaborar sus emociones y afectos. Se trata de un proceso complejo que puede durar mucho tiempo y en el que influyen de manera positiva factores como el hecho de vivir en un contexto que ofrece oportunidades para una buena inserción laboral, social, cultural y económica.

\section{El nuevo contexto y los planes hacia el futuro}

Vivir en Buenos Aires, para muchas de las entrevistadas, representa un cambio notable en diversos aspectos de sus vidas. Casi todas las mujeres de este estudio se han integrado en el mundo laboral con cierto éxito, y aunque para algunas el proceso ha sido más largo y laborioso, al final de cuentas les he permitido cumplir con los objetivos de su proyecto migratorio e ir avanzando en su trayectoria profesional. Económicamente, si bien algunas de ellas ya trabajaban en Colombia antes de emigrar a Argentina, en Buenos Aires obtienen salarios que les permiten cultivar sus intereses, también los de carácter lúdico y cultural, y en últimas afirmarse a sí mismas. Aunque algunas han perdido en un comienzo y en alguna medida su bienestar socio-económico, ello no parece representar una renuncia insuperable ya que al final han conseguido independizarse completamente de la familia y, por ende, de muchos de sus condicionamientos.

En el nuevo contexto se notan cambios también en aspectos más íntimos de las mujeres, como es la feminidad y la expresión de la vida sexual. La migración e inserción en un ambiente diferente y que ofrece diversas oportunidades les da a muchas de las entrevistadas la posibilidad "de ser", más allá de los estereotipos con los que han crecido, y de entender que pueden vivir sus propios sueños con menos limitaciones y constricciones. En muchos casos representa la libertad de no ser "nadie" y de vivir la propia vida sin tener que cumplir con comportamientos preestablecidos.

La vida en Buenos Aires - un contexto que perciben como más abierto-, lejos del control de la sociedad de origen y con una distancia que les protege su 
intimidad, permite que muchas mujeres puedan vivir más libremente su vida y orientación sexual, que en Colombia parece estar muy determinada por roles y expectativas sociales. La estadía en Buenos Aires podría llegar a ser entendida por ellas mismas como un pasaje hacia una vida de persona adulta libre, pasaje que les resultaría más dificultoso encarar en sus sociedades de origen.

Es de notar además algunas entrevistadas están muy satisfechas porque viven en un entorno jovial donde no perciben discriminaciones de ningún tipo, y además es un ambiente que consideran seguro, en el que pueden vivir tranquilas $\sin$ las angustias de la inseguridad típica de la sociedad colombiana. De hecho, creen que la situación en Buenos Aires es manejable, y que las precauciones que tienen que tomar son normales, siendo fácil y poco exigente acostumbrarse a ellas.

Esto no significa, ciertamente, que no echen de menos muchos aspectos de su cultura de origen, como la música, el baile y la comida. De la misma manera, algunas añoran los paisajes, la naturaleza, la ciudad y algunos espacios físicos que representan una suerte de memoria social y personal. En este sentido, se percibe un fuerte sentimiento de añoranza que se refiere a un espacio geográfico pero también a los "lugares en el tiempo", con imágenes profundamente evocadoras de la vida y la trayectoria individual de cada una. Estas se convierten en un componente importante para la configuración de su identidad, que se alimenta de la relación dialéctica entre referentes culturales diversos.

Aunque algunas de las entrevistadas afirman que a veces no entienden los códigos culturales de comportamiento de los argentinos, en especial en la esfera amorosa, y a veces les cuesta amoldarse, tienen en Buenos Aires amigos colombianos, argentinos y de otras nacionalidades. Están inmersas en un ambiente multicultural y también "transnacional", ya que viajan a Colombia más o menos regularmente y algunas son visitadas por sus familias (en especial por las madres) y, en ocasiones, sus por amigos. Es usual, además, que mantengan contactos con los amigos en Colombia con quienes tienen vínculos de mayor cercanía, aunque el estar lejos determina la configuración de diferencias más o menos acentuadas y significativas en sus modos de ser y de vida.

En lo que concierne a la esfera social, las entrevistadas logran aprovechar lo que la ciudad brinda más allá de la vida laboral. Sienten una fuerte atracción por el fenómeno urbano y por la vida cultural accesible y hasta gratuita, así como por la vida nocturna y su oferta cultural y de esparcimiento, por las diversas oportunidades de disfrute del tiempo libre. Coinciden en que en Buenos Aires nadie juzga a los demás por su aspecto, y se sienten muy a gusto por la ausencia del control social, que en muchos casos las oprimía en su lugar de origen.

Todos estos elementos los perciben como positivos, siendo determinantes para que algunas de las entrevistadas quieran quedarse en Argentina de manera 
definitiva a pesar de la crisis económica del país y de las mayores dificultades que esto acarrea para los extranjeros. El querer quedarse es muy usual entre quienes tienen una pareja estable con la cual se sienten a gusto y con la que se ven proyectadas hacia el futuro. Es de notar, sin embargo, que hay quienes están solas en Buenos Aires pero que igual pretenden establecerse allí.

Otras mujeres, a pesar de haber encontrado trabajo y de tener ciertas posibilidades de mejorar profesionalmente, tienen planes de quedarse sólo temporalmente. Muchas de ellas tienen una sed enorme de conocimiento y quieren seguir viajando para conocer gente diferente, otras realidades e ir acumulando competencias y experiencias. El deseo de explorar y de aventura está muy presente, y es frecuente que estar en Buenos Aires sea asumido como algo pasajero, útil para seguir hacia nuevos rumbos. La idea de retornar a Colombia es algo que algunas contemplan sólo como un proyecto a largo plazo.

\section{Conclusiones}

Como se ha podido ver, los procesos migratorios de las colombianas con alta calificación entrevistadas para el presente trabajo muestran características diferentes entre sí, aunque revelan regularidades que los acomunan y que determinan en general recorridos positivos para su desarrollo personal. Ello sin negar que están permeados por sentimientos de nostalgia, pérdida, renuncia y dolor, como es normal que ocurra en este tipo de procesos.

En las motivaciones que mueven a las entrevistadas a migrar se advierte una matriz emocional, aunque frente a la familia las jóvenes entrevistadas no siempre expresan abiertamente estos aspectos. Muchas de ellas utilizan en cambio la excusa o la "motivación de segundo nivel" de emigrar por motivos de estudio y laborales, lo que facilita la aprobación del grupo familiar y el que este no obstaculice su proyecto. De hecho, la migración de estas mujeres se inscribe en el marco de una cultura que considera el estudio y el trabajo en el extranjero como un elemento cardinal para la "superación" del individuo y para su "crecimiento" personal, profesional y económico.

Es de notar que, si se observa en profundidad, en las razones para emigrar de las entrevistadas subyace el deseo de conocer nuevos mundos y formas de ser, distintos de los propios. Este deseo está relacionado con otra motivación de orden psicológico que concierne la resolución del estrés que comporta la definición de la autonomía, la emancipación emocional y familiar, y la definición de la identidad. Efectivamente, mediante la migración, las jóvenes ponen distancia geográfica con sus familias, lo que las ayuda a manejar vínculos familiares con fuerte tracción endogámica y el impacto emocional que ello acarrea en sus vidas.

No obstante esta situación que se define como estresante, las entrevistadas demuestran capacidad de agencia. Viven activamente el proyecto migratorio, 
tomando decisiones que las ayudan a lograr independencia económica y a asumir conductas fuera de los esquemas sociales de su país de origen y de roles tradicionales y patriarcales para las que muchas han sido educadas.

Se puede observar además cómo logran manejar y superar una fase de su existencia en la que deben afrontar diversas transformaciones a nivel psíquico y emocional. Si bien durante el proceso migratorio las entrevistadas se nota una prolongación de la etapa "adolescente" en parte por las muchas novedades y cambios que deben afrontar en la sociedad de destino, la estadía fuera del país de origen puede ser entendida como un pasaje hacia la vida de adulto libre. Un tránsito que les resultaba dificultoso encarar en el contexto de origen por las constricciones sociales y familiares. De hecho, durante la migración las entrevistadas revierten algunos esquemas sociales de su lugar de origen, reelaboran problemas familiares y algunas veces solucionan conflictos de orden emotivo.

Es un hecho que la migración se configura como una búsqueda de nuevas posibilidades de vida. Las entrevistadas quieren ampliar su campo experiencial hacia situaciones que no están en la paleta de conductas habituales. Aprovechan de la vida cultural y algunas de ellas están abiertas también a desarrollar en su tiempo libre actividades de carácter cultural que nunca habían experimentado y que nada tienen que ver con el campo laboral al que se han dedicado.

La búsqueda se refleja también en la dimensión afectiva y en el área del desarrollo de la sexualidad. Si bien no lo expresen abiertamente, muchas entrevistadas buscan relaciones románticas novedosas. Aunque no todas logran establecer relaciones afectivas estables, es cierto que entran en contacto con modos de vivir la sexualidad que resultan ser más flexibles y libres, reflejo de una sociedad abierta -especialmente en Buenos Aires- en donde es más fácil experimentar dichas relaciones y las diversas orientaciones sexuales. A este propósito hay que recordar que en Argentina desde el año $2010^{36}$ está vigente el matrimonio homosexual y que está equiparado al matrimonio heterosexual, así como que hay también libertad de cambio de sexo.

En el grupo de mujeres entrevistadas no se observa sufrimiento psíquico, al contrario de lo que ocurre en otros grupos de migrantes ${ }^{37}$; no padecen particularmente el choque cultural, pues llegan a un contexto que sienten cercano y que no perciben hostil porque en su proceso migratorio no tienen que enfrentarse a comportamientos prejuiciosos ni perciben discriminación de parte de los ciudadanos argentinos. Entre las entrevistadas no se hallan

\footnotetext{
${ }^{36}$ Es el primer país en América Latina en haber reconocido y legalizado este tipo de unión, y el décimo a nivel mundial.

${ }^{37}$ COUTO, Diana. Exiliación de la migración. Una mirada psicopatológica al duelo migratorio patológico.
} 
tampoco vestigios relevantes de estrés aculturativo, categoría analítica que hace referencia a la discriminación percibida, a la nostalgia y al choque cultural ${ }^{38}$.

Aunque no todas las mujeres viven la migración de la misma manera, entre las entrevistadas se notan procesos de emancipación y subversión de los roles de género, que aun siendo poco lineares y a veces discordantes, son significativos en el cuadro de la trayectoria personal de cada una de las mujeres que participaron en el estudio. Se percibe cómo se hacen cargo en primera persona de su vida, en una búsqueda constante de sí mismas, con implicaciones diferentes en las diversas dimensiones del ser, según los casos y según los obstáculos que encuentran. Cambios que, como se pudo observar, derivan de su historia individual, de sus recursos personales o también de las diferentes experiencias que viven durante el proceso migratorio.

Es innegable la influencia positiva del contexto social que las acoge de manera afable y en donde este grupo de mujeres calificadas profesionalmente tienen oportunidades de integrarse laboralmente; y donde, además, su proveniencia no es motivo de segregación, sino que en algunos casos puede constituir hasta una ventaja. Influye igualmente de manera positiva el que las mujeres puedan mantener los lazos con el país de origen, con viajes más o menos frecuentes y con una comunicación intensa con algunas personas que para ellas representan figuras importantes en la dimensión emocional de su existencia. Las relaciones familiares transnacionales, aun presentando un carácter dialéctico por representar ser un centro de referencia emocional y también ser un canal de transmisión de conflictos familiares, se configuran como una oportunidad para vivir la migración no como una pérdida, sino como el fruto de una elección fundamentalmente positiva y enriquecedora en la trayectoria vital.

Por último y para concluir, el proceso migratorio en el caso específico estudiado, por la edad y las circunstancias en que se realiza, se podría entender como una fase decisiva para finalizar el proceso de la adolescencia en el que las jóvenes resuelven de una manera o de otra una serie de transformaciones de orden psíquico, social y cultural, como la búsqueda de sí mismas, la emancipación económica, familiar y social, la re-jerarquización de los vínculos afectivos y la construcción de nuevos vínculos, la definición de aspectos de la identidad y también la concepción de planes y proyectos para el futuro.

\section{Bibliografía}

AMBROSINI, Maurizio. Dentro il welfare invisibile: aiutanti domiciliari immigrate e assistenza agli anziani. Studi Emigrazione, ano XLII, n. 159, 2005, p. 561-595. BASH, Linda; GLICK-SCHILLER, Nina; SZANTON-BLANC, Cristina. Toward a Transnational Perspective on Migration. New York: Academy of Sciences Nueva York, 1992.

${ }^{38}$ COLLAZOS, Francisco et alii. Estrés aculturativo y salud mental en la población inmigrante. 
BERTAUX, Daniel. Racconti di vita. La prospettiva etnosociologica. Milán: Franco Angeli, 1999.

BOYD, Monica; GRIECO, Elizabeth. Women and migration: Incorporating gender into international migration theory. Migration Information Source. 2003. Disponible en: <http://www.migrationinformation.org/Feature/display. cfm? id=106>. Consultado el 03.05.2016.

BOUCHER, Anna. Skill, migration and gender in Australia and Canada: The case of gender-based analysis. Australian Journal of Political Science, v. 42, n. 3, 2007, p. 383-401.

BRYCESON, Deborah; VOURELA, Ulla (eds.). The transnational family. New European frontiers and global networks. Londres \& Nueva York: Berg, 2002.

CIURLO, Alessandra. Nueva política migratoria colombiana: el actual enfoque de inmigración y emigración. Revista Internacional de Cooperación y Desarrollo, v. 2, n. 2, julio/diciembre, 2015, p. 205-242.

CIURLO, Alessandra. La migración femenina y los cambios en las relaciones de género en las familias: el caso de las transmigrantes colombianas en Italia. Oasis, v. 21, 2015, p. 55-79.

CIURLO, Alessandra. Migración colombiana hacia Italia a la luz del género y la familia transnacional. Bogotá: Universidad Antonio Nariño, Fundación Esperanza, 2013.

CALVELO, Laura; COUTO, Diana; OSORIO, María Emilia. Migración internacional y Salud en Argentina. El impacto de las Migraciones en el sistema de Salud. Buenos Aires: EDUNTREF, 2016.

COLLAZOS, Francisco; QURESHI, Adil; ANTONÍN, Montserrat; TOMÁS SÁBADO, Joaquín. Estrés aculturativo y salud mental en la población inmigrante. Papeles del Psicólogo, v. 29, n. 3, septiembre-diciembre, 2008, p. 307-315.

COUTO, Diana. Exiliación de la migración. Una mirada psicopatológica al duelo migratorio patológico. Revista IDEIA.Net, año 4, n. 6, 2013, p. 23-33. Disponible en: < http://www.diversidadcultural.net/articulos/nro006/06-02-diana-couto. pdf $>$. Consultado el 27.07.2016.

DI LORENZO, Delfina. El Estado argentino frente al fenómeno de la migración de estudiantes universitarios de origen latinoamericano (2003-2011). Primeras Jornadas Internacionales Sociedad, Estado y Universidad. Mesa 17. Post neoliberalismo: el debate en torno a los modelos, estrategias y modos de desarrollo en América Latina. Mar del Plata - Argentina, 30 de noviembre, 1 y 2 de diciembre, 2011.

DOCQUIER, Frédéric; MARFOUK, Abdeslam; SALOMONE, Sara; SEKKAT, Khalid. Are skilled women more migratory than skilled men? IRES Discussion papers, $\mathrm{n}$. 21, 2009, p. 1-30.

EREL, Umut. Skilled migrant women and citizenship. In MOROKVASIC, Mirjana; EREL, Umut; SHINOZAKI, Kyoco (eds.). Crossing Borders and Shifting Boundaries, v. 1, Gender on the Move, 2003, p. 261-83. 
GRIGOLEIT, Grit. Making a career? The integration of highly skilled female migrants into the German job market. In APPAM International Conference. Migration: A World in Motion a Multinational Conference on Migration and Migration Policy. Maastricht, Netherlands, February 18-20, 2010.

HIGUERA, Diego et alii. Aportes para la comprensión de la llegada de estudiantes colombianos a las universidades argentinas: Construcción de un estudio exploratorio. I Jornadas Interdisciplinarias de jóvenes Investigadores en Ciencias Sociales. IDAES/UNSAM. Mesa 4 Migraciones: Identidad y política. Ciudad Autónoma de Buenos Aires - Argentina, 8, 9 y 10 de mayo, 2013.

HOCHSCHILD, Arlie R. Global care chains and emotional surplus value. In HUTTON, Will; GIDDENS, Anthony (eds.). On the Edge. Living with Global Capitalism. London: Vintage, 2001, p. 130-146.

HONDAGNEU SOTELO, Pierrette; AVILA, Ernestine. I'm here, but I'm there: The meaning of Latin transnational motherhood. Gender and Society, n. 11, 1997, p. 548-565.

KABEER, Naila. Benevolent dictators, maternal altruists and patriarchal contracts: Gender and household economics. In KABEER, Naila. Reversed Realities: Gender hierarchies in development thought. Londres: Verso, 1994.

KOFMAN, Eleonore. Gender and skilled migration in Europe. Cuadernos de Relaciones Laborales, v. 30, n. 1, 2012, p. 63-89.

LAZARUS, Richard S. Stress and emotion. A new synthesis. Nueva York: Springer, 1999. MELELLA, Cecilia. Migraciones emergentes hacia la Argentina: colombianos y ecuatorianos. Breve panorama y estrategias de inserción cultural. Si Somos Americanos, v. 14, n. 2, Dic, Santiago de Chile, 2014.

MERLA, Laura. La circulación de cuidados en las familias transnacionales. Revue CIDOB d'Afers Internacionals, n. 106-107, septiembre, 2014, p. 85-104.

OIM. La migración colombiana en Argentina. Buenos Aires: Consulado de Colombia en Buenos Aires - Universidad Nacional de Tres de Febrero - Instituto de Políticas Migratorias y Asilo (IMPA) - Organización Internacional para las Migraciones (OIM) Oficina País para Argentina, 2016.

OIM. Panorama migratorio de América del Sur. Buenos Aires: OIM, 2012.

OIM. Perfil migratorio de Colombia. Bogotá: OIM, 2012.

OIM. Perfil migratorio de Colombia. Bogotá: OIM, 2010.

OSO, Laura. L'immigration en Espagne des femmes chefs de famille. Les cahiers du CEDREF, 8-9, 2000. Disponible en: < http://cedref.revues.org/191>. Consultado el 20.05.2016.

PARELLA, Sònia. La maternidad a distancia de las empleadas domésticas latinoamericanas en España. La vulneración del derecho a la vida familiar en el contexto de la internacionalización de la reproducción. In GIRÓ, Joaquín (ed.). El género quebrantado. Sobre la violencia, la libertad y los derechos de la mujer en el nuevo milenio. Madrid: Catarata, 2005, p. 238-273. 
PARREÑAS, Rachel. Servants of globalization: Woman migration and domestic work. Stanford University Press, 2001.

PUENTE, M. Cuando migrar es la opción. Estudiantes colombianos en Argentina: motivos y experiencias en sus trayectorias migratorias y estudiantiles. Tesis de Maestría, Universidad de Buenos Aires, Argentina, 2011.

RAGHURAM, Parvati. Situating women in the brain drain discourse: Discursive challenges and opportunities. In STALFORD, Helen; CURRIE, Samantha; VELLUTI, Samantha (eds.). Gender and migration in 21st century Europe. Aldershot: Ashgate, 2009, p. 85-106.

RAGHURAM, Parvati. The difference that skills make: Gender, family migration strategies and regulated labour markets. Journal of Ethnic and Migration Studies, v. 30, n. 2, 2004, p. 303-323.

RIAÑO, Yvonne; PIGUET, Etienne. International student migration. In WARLF, Barney (ed.). Oxford Bibliographies in Geography. Nueva York: Oxford University Press, 2016, p. 1-29.

RIAÑO, Yvonne. Minga biographic workshops with highly skilled migrant women: Enhancing spaces of inclusion. Qualitative Research, Special Issue on Feminist Participatory Methodologies, Sage, 2015, p. 1-13.

RIAÑO, Yvonne; BAGHDADI, Nadia; WALST WATER, Doris. Les migrantes qualifiées et leurs possibilités d'intégration professionnelle en Suisse. Berna: Instituto de Geografía - Universidad de Berna, 2008.

SAUTU, Ruth (comp.). El método biográfico. La reconstrucción de la sociedad a partir del testimonio de los actores. Buenos Aires: Editorial de Belgrano, 1999.

SISTEMA UNIVERSITARIO ESTATAL. Desfinanciamiento de la educación superior en Colombia. Pereira: Universidad Tecnológica de Pereira, 2012.

Recibido para publicación en 19.09.2016

Aceptado para publicación en 18.10.2016 Received for publication in September 19, 2016 Accepted for publication in October $18^{\text {th }}, 2016$

ISSN impresso 1980-8585

ISSN eletrônico 2237-9843

http://dx.doi.org/10.1590/1980-85852503880004810 